\title{
OBSERVATIONS HISTOLOGIQUES SUR LES CAPSULES INDUITES PAR CONTORTYLENCHUS DIPLOGASTER V. LINS. (NEMATODA : ALLANTONEMATIDAE) dans le tissu adipeux d'Ips sexdentatus Boern. (Coleoptera : Scolytidae)
}

\author{
F. LIEUTIER* \\ Collaboration technique : M. Jastrabsky et P. Bonnafe
}

RÉSUMÉ. L'évolution des capsules induites par la présence de Contortylenchus diplogaster dans le tissu adipeux d'Ips sexdentatus est suivie par une étude histologique depuis la nymphose du Scolyte jusqu'à sa ponte.

Au début du stade nymphal de l'insecte, la femelle parasite de $C$. diplogaster n'a pas encore pondu et les noyaux de la capsule qui l'entoure sont groupés par tétrades; certains présentent un état de dégénérescence avancée. Ces observations suggèrent que la capsule syncytiale résulterait, en plus de fusions cellulaires, d'une multiplication amitotique des noyaux, suivie d'une dégénérescence de certains d'entre eux. A la fin du stade nymphal, les noyaux, séparés les uns des autres, sont répartis à la périphérie de la capsule. La ponte du nématode a lieu quand l'hôte est adulte. Après développement des larves à l'intérieur de la capsule, celle-ci finit par se rompre en général après que le scolyte ait attaqué un nouvel arbre-hôte. Les larves quittent alors l'insecte et se répandent dans les galeries.

Le développement parasitaire de $C$. diplogaster s'effectue toujours en totalité dans le tissu adipeux du Scolyte.

\section{Histological observations on the capsules induced by Contortylenchus diplogaster v. Lins (Nematoda : Allantonematidae) in the fat body of Ips sexdentatus (Coleoptera : Scolytidae).}

SUMMARY. The evolution of the capsules induced by $C$. diplogaster in the fat body of $I$. sexdentatus is followed by histological methods from the pupal stage to the oviposition of the host.

Early in the pupal stage of the insect, the parasitic female of $C$. diplogaster has not oviposited yet and the nuclei of the capsule that surrounds it are gathered in tetrads; some of these nuclei are in a well forwarded degenerescence. These observations suggest that the syncytial capsule should result both from an amitotic division of the nuclei followed by a degenerescence of some of them and from a fusion of cells. Late in the pupal stage, the nuclei are set apart from each other and distributed at the periphery of the capsule. The oviposition of the nematode takes place when the host is an adult. After the development of the larvae has been made into the capsule, this one ends in breaking, generally when the bark beetle has attacked a new host-tree. Then, the larvae of the nematode leave the insect and invade the galleries.

The parasitic development of $C$. diplogaster always takes place quite in the fat body of the beetle.

* Laboratoire de Zoologie, Institut National Agronomique, 16 rue Claude-Bernard, F 75231 Paris Cedex 05.

Accepté le 2 août 1983. 
Nous avons précédemment signalé que, lors d'une dissection de l'insecte-hôte Ips sexdentatus, les femelles parasites de Contortylenchus diplogaster et leur progéniture étaient souvent observées à l'intérieur de capsules provenant du tissu adipeux (Lieutier et Seureau, 1981). Ces capsules sont le résultat d'une " hypertrophie des cellules adipeuses et d'une rupture de leur membrane cellulaire sous l'action du nématode parasite, aboutissant à la formation d'un syncytium. "

Toutefois, cette réaction d'encapsulement semblait ne concerner que certaines femelles de $C$. diplogaster, les autres étant libres dans l'hémocoele de l'hôte. Il nous a paru alors intéressant, d'une part de déterminer quels pouvaient être le mode de formation et l'évolution des capsules, d'autre part, de préciser si les localisations des nématodes dans les capsules ou dans l'hémocoele pouvaient correspondre à des étapes différentes de la maturation des parasites dans l'hôte ou simplement à la rupture des capsules extrêmement fragiles lors de la dissection. La présente note a pour objet d'apporter des informations sur ces divers points.

Les cycles du parasite et de l'hôte ont été décrits précédemment (Lieutier 1979, Lieutier et Seureau, 1981).

\section{1 - Méthodes et techniques.}

Les insectes sont suivis depuis le stade nymphal jusqu'à la ponte. Les adultes sont placés dans le fixateur de Carnoy; les pattes, les ailes et la tête sont retirées, puis les insectes sont coupés en deux au niveau de la partie postérieure du prothorax, de façon à séparer le thorax de l'abdomen. La fixation dure 4 heures. Après déshydratation, l'inclusion à la paraffine est réalisée suivant la technique habituelle (Martoja et Martoja, 1967). Les coupes d'une épaisseur de 5 à $7 \mu \mathrm{m}$, sont colorées par le glychemalun et l'érythrosine. Chez les nymphes, les capsules sont obtenues par dissection de l'insecte dans la solution de Ringer, puis fixées pendant 2 heures dans le Carnoy ou le Bouin alcoolique; les coupes sont colorées par l'Azan de Heidenhaim ou le glychemalun-érythrosine.

\section{2 - Résultats et interprétation.}

1 - Formation et Évolution des capsules.

Quand l'hôte est au stade nymphal, sa contamination est récente ; les femelles de Contortylenchus n'ont pas terminé leur maturation et n'ont pas encore pondu. La capsule adipeuse est de petite taille $(70 \times 300 \mu \mathrm{m})$ et les noyaux se trouvent groupés en son centre par tétrades ( fig. 1 et 2). L'examen détaillé d'une tétrade montre que les quatre noyaux sont reliés entre eux en un même point (fig. 3) ; le plus souvent, l'un des noyaux se trouve dans un état de dégénérescence avancé. L'enveloppe nucléaire des trois autres se distingue bien, du moins en dehors de la zone qui les relie ; la chromatine est dispersée dans tout le nucléoplasme ; les nucléoles sont bien visibles. 
Ces images autorisent à penser que les noyaux adipeux de la capsule sont en division. L'absence de chromosomes différenciés et de figures de mitose, ainsi que la persistance de l'enveloppe nucléaire, suggèrent qu'il pourrait s'agir d'amitoses. La présence simultanée de plusieurs tétrades dans la même capsule pourrait être due à une division simultanée de certains noyaux et (ou) à un blocage des amitoses. L'existence même des tétrades est néanmoins curieuse. Elle tend à montrer que le noyau initial s'est divisé directement en quatre noyaux fils. En effet, il s'agissait de deux divisions successives, le point d'attache ne serait sans doute pas le même pour les quatre noyaux.

Ces résultats suggèrent que le syncytium adipeux induit par la présence des nématodes provient non seulement de la lyse des membranes cellulaires, comme l'indique la présence de restes de membrane entre les noyaux (Lieutier et Seureau, 1981), mais encore d'une multiplication amitotique des noyaux.

Comme l'ont fait Poinar et Hess (1974) pour Abbreviata caucasica, nématode parasite de Blatella germanica, nous pouvons rapprocher nos observations de celles qui ont été effectuées chez certains nématodes parasites de végétaux (Bird, 1974, Endo, 1975, Jones, 1981). Il est probable en particulier que la transformation du tissu adipeux en syncytium s'effectue sous l'action d'une secrétion du nématode, certainement par la femelle parasite. Dans le cas de $C$. diplogaster, nous remarquerons toutefois que la sécrétion ne semble pas capable de traverser la membrane basale du tissu adipeux, puisque l'hypertrophie reste limitée aux seuls lobes parasités. Par ailleurs, si le résultat de l'action des nématodes est comparable à ce qui se passe chez les végétaux, les modalités apparaissent différentes. Ainsi chez les végétaux parasités (Jones, 1981) les espèces du genre Meloidogyne induisent la formation de cellules géantes à plusieurs noyaux par mitoses successives de ceux-ci sans cytodiérèse, mais on n'observe pas de fusion entre cellules voisines. Le genre Heterodera induit par contre des fusions cellulaires qui contribuent de façon importante à la formation du syncytium et il ne semble pas que des mitoses interviennent. Le genre Nacobbus induit simultanément des fusions cellulaires et des mitoses, mais ces dernières paraissent ne concerner que des cellules qui ne sont pas incorporées dans le cyncytium. Chez les insectes parasités par $C$. diplogaster en revanche, le syncytium semble être formé à la fois par fusion cellulaire et division des noyaux.

Après division, un noyau de chaque tétrade dégénérerait, les autres finiraient par se séparer. Ce point de vue est confirmé par l'observation, chez les nymphes jeunes, de noyaux isolés dont la membrane présente une structure pouvant correspondre à la trace du point d'attache (fig. 3).

La structure typique de la capsule apparaît juste avant la mue imaginale de l'insecte. A ce moment en effet, les noyaux se trouvent à la périphérie, le cytoplasme est développé (fig. 4). Quand le scolyte est au stade adulte, la femelle de Contortylenchus pond; les œufs éclosent et, durant la maturation de préessaimage de l'hôte, les larves de nématodes envahissent progressivement toute la capsule faisant disparaître le cytoplasme et les noyaux (fig. 5). En fin d'évolution et au moment de l'essaimage, il ne subsiste plus de la capsule qu'une très fragile et fine membrane 
constituant un sac bourré de larves (Lieutier et Seureau, 1981). Celui-ci finit par se rompre libérant son contenu dans l'hémocoele de l'hôte (fig. 6).

Après l'éclatement des capsules, quand le scolyte est parvenu sur un nouvel arbre et a commencé à pondre, les larves de $C$. diplogaster quittent leur hôte. Nous n'avons pas pu suivre directement le départ des nématodes, mais nous avons pu observer, sur coupe histologique d'une femelle d'I $p s$ parasitée, le début du passage à travers la paroi de l'oviducte de l'insecte, de larves de $C$. diplogaster récemment libérées par la rupture d'une capsule (fig. 7). Rühm (1956), pour l'ensemble des Contortylenchus qu'il a étudiés (dont $C$. diplogaster parasite d'Ips typographus et d'Ips cembrae), Massey $(1962,1974)$ et Nickle $(1963,1974)$ pour Contortylenchus elongatus indiquent que les larves de ces nématodes sortent par l'anus de leur hôte, après avoir transité par son tube digestif. Il est donc probable que les deux voies, digestive et génitale, peuvent être empruntées par les larves de $C$. diplogaster pour gagner les galeries de leur hôte. Mac Guidwin et coll. (1980) signalent également le passage par les voies génitales, en plus du tube digestif, pour Contortylenchus brevicomi chez Dendroctonus frontalis.

FIG. I. - Coupe longitudinale d'une capsule adipeuse contenant une femelle parasite de $C$. diplogaster, dans une nymphe d'I. sexdentatus en début de développement. c : cytoplasme ; f : femelle parasite; n : noyau; t : tétrade. (Fixation Carnoy; coloration glychemalun-erythrosine).

Pour cette figure comme pour les suivantes, la longueur du trait d'échelle est exprimée en microns.

FIG. 2. - Coupe transversale d'une capsule adipeuse contenant une femelle parasite de $C$. diplogaster, dans une nymphe d'I. sexdentatus en début de développement. c : cytoplasme ; f : femelle parasite; $\mathrm{n}$ : noyau; t : tétrade. (Fixation Carnoy; coloration glychemalunérythrosine).

FIG. 3. - Coupe d'une capsule dans la région des tétrades chez une nymphe d'I. sexdentatus en début de développement. c : cytoplasme; $d$ : noyau en dégénérescence ; n : noyau. La flèche indique une structure pouvant correspondre à une trace de point d'attache. (fixation Carnoy; coloration glychemalun-erythrosine).

Fig. 4. - Capsule contenant une femelle parasite de $C$. diplogaster dans une nymphe d'I. sexdentatus peu de temps avant la mue imaginale. $\mathrm{c}:$ cytoplasme ; $\mathrm{f}$ : femelle parasite ; $\mathrm{n}:$ noyau (fixation Bouin ; coloration Azan).

Frg. 5. - Capsule contenant une femelle parasite de $C$. diplogaster et sa descendance dans un adulte d' $I$. sexdentatus en maturation de préessaimage. $\mathrm{f}$ : femelle parasite ; 1 : larves de nématode ; $\mathrm{n}$ : noyau de la capsule; $\mathrm{t}$ : tissu adipeux. (fixation Carnoy ; coloration glychemalunérythrosine).

FIG. 6. - Capsule éclatée dans un adulte d'I. sexdentatus. 1 : larves de nématodes; les flèches indiquent les restes de membrane de la capsule (fixation Carnoy; coloration glychemalunerythrosine).

Fig. 7. - Passage d'une larve de $C$. diplogaster à travers l'oviducte d' $I$. sexdentatus; $1:$ larves de nématodes ; lu : lumière de l'oviducte ; p : paroi de l'oviducte. (fixation Carnoy ; coloration glychemalun-erythrosine). 

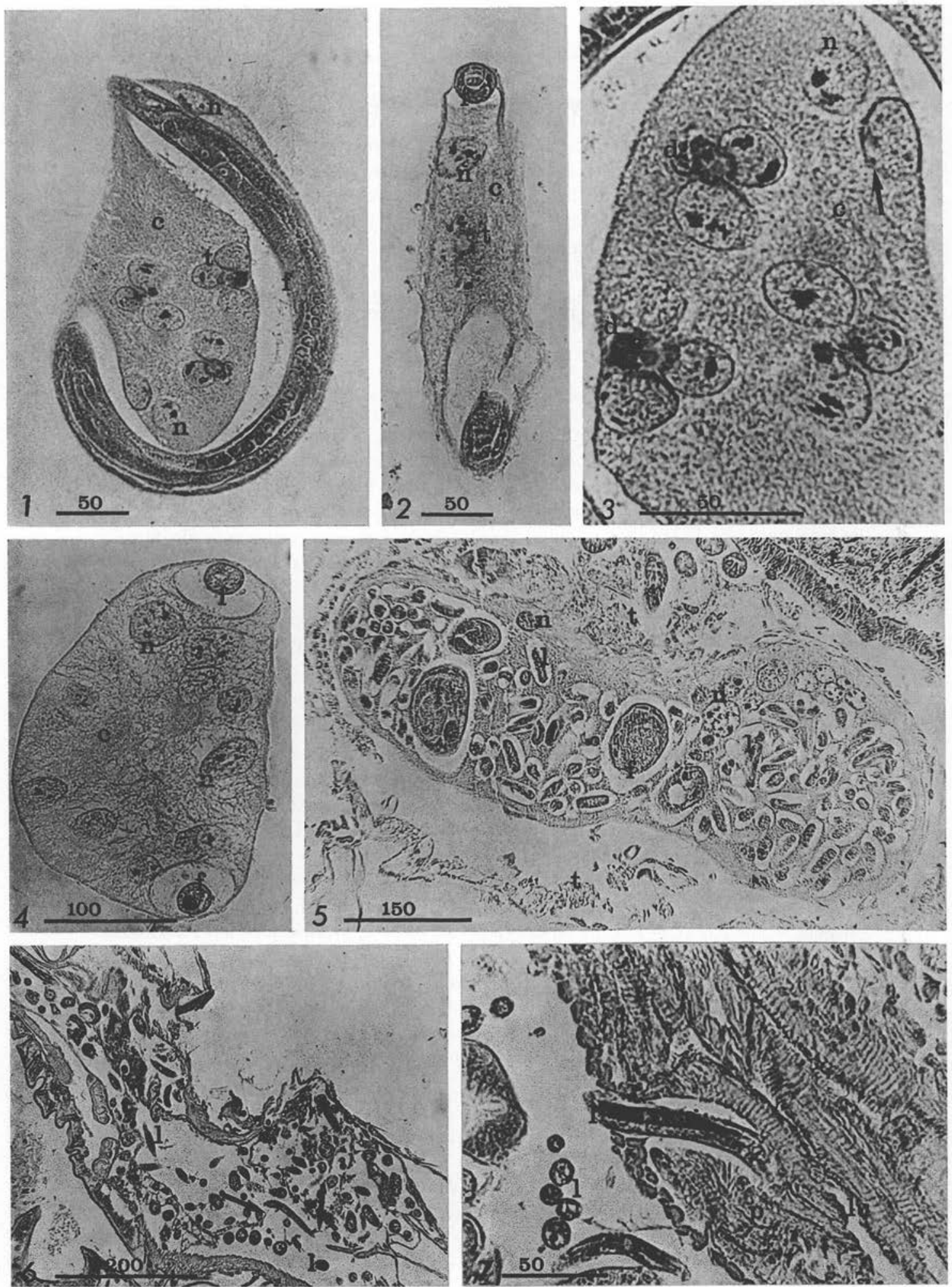
L'évolution des capsules décrite ici pour $C$. diplogaster correspond au type le plus fréquemment observé; certaines capsules font cependant exception. Ainsi, $4 \%$ environ des femelles parasites n'ont toujours pas pondu au moment de l'essaimage de leur hôte (Lieutier, 1979). Dans d'autres cas au contraire, l'évolution est en avance et les capsules se rompent dès l'essaimage. Dans ces conditions, il n'est pas impossible que la libération massive et brutale de larves dans la cavité générale du Scolyte provoque un choc physiologique et perturbe fortement celui-ci, d'autant plus qu'il se trouve alors à une période de grands besoins énergétiques. Peut-être faut-il voir là un facteur responsable de la mort de certains Ips pendant leur vol (Lieutier, 1979).

\section{2 - Existence de formes non encapsulées de C. diplogaster dans les insectes.}

Pendant toute la maturation de préessaimage de l'insecte, les coupes histologiques d'individus entiers montrent toujours les Contortylenchus localisés à l'intérieur de leurs capsules adipeuses, jamais libres dans l'hémocoele (fig. 5). Bien qu'il arrive fréquemment que la dissection d'un Ips sexdentatus en cours de maturation de préessaimage libère des milliers d'œufs et de larves accompagnés de femelles parasites, cet événement est donc accidentel et correspond en fait à la rupture des capsules très fragiles lors de la dissection.

A partir de l'essaimage et surtout pendant la maturation de ponte du Scolyte, les coupes histologiques d'insectes entiers montrent des femelles et des larves de nématodes libres dans la cavité générale de leur hôte. Dans ce cas elles proviennent de la rupture de capsules arrivées en fin d'évolution, ainsi que le confirme l'observation de débris de capsules sur les coupes ( $f$ g. 6).

C. diplogaster effectue donc la quasi-totalité de son développement parasitaire dans le tissu adipeux de son hôte, depuis le début de la maturation de la femelle parasite jusqu'à la fin de la croissance des larves parasites. Cette localisation constitue une originalité dans la famille des Allantonematidae, puisque les adultes parasites de toutes les espèces de cette famille sont considérés comme libres dans la cavité générale de leur hôte où ont lieu la ponte et le développement des larves (Fuchs 1915 Rühm, 1956, Welch 1965, Massey 1974, Nickle 1974, Poinar 1975). Dans ces conditions, on peut se demander si le type de développement et la localisation de $C$. diplogaster chez I. sexdentatus ne sont pas ceux de tous les Contortylenchus. Des études sur ce sujet sont évidemment nécessaires, mais le fait que l'on ait pu observer des capsules semblables à celles d'I. sexdentatus lors de dissections d'I $p$ s accuminatus (Lieutier, non publié) nous fait penser que ce doit être au moins le cas pour Contortylenchus acuminati.

Remerciements. L'auteur remercie Monsieur C. Chararas, Directeur de recherches au CNRS, Membre de l'Institut et Monsieur le Professeur Pesson (Institut National Agronomique) de leurs précieux conseils, ainsi que C. Seureau (Université Paris VI) d'avoir bien voulu relire et critiquer le manuscrit. 


\section{BIBLIOGRAPHIE}

Bird A. F. : Plant response to Root-knot nematodes. Ann. Rev. Phytopathol., 1974, I2, 69-85.

Endo B. Y. : Pathogenesis of nematode infected plants. Ann. Rev. Phytopathol., 1975, 13, $213-238$.

Fuchs G. : Die Naturgeschichte der Nematoden und einiger anderer parasiten I. des Ips typographus. 2. des Hylobius abietis. Zool. Jahrb. (Syst.), I91 5, 38, 109-222.

Jones M. G. K. : Host Cell responses to endoparasitic nematode attack : structure and function of giant cells and syncytia. Ann. Appl. Biol., 1981, 97, 353-372.

Lieutier F. : Le parasitisme d'Ips sexdentatus (Coleoptera Scolytidae) par Contortylenchus diplogaster (Nematoda: Allantonematidae). Revue Nematol., 1979, 2, 143-151.

Lieutier F., Seureau C. : Encapsulement cellulaire de Contortylenchus diplogaster V. lins. (Nematoda : Allantonematidae) dans le tissu adipeux d'I ps sexdentatus Boern., (Coleoptera : Scolytidae). Ann. Parasitol. Hum. Comp., I981, s6, 607-612.

Mac Guidwin A. E., Smart G. C., Allen G. E. : Redescription and life history of Contortylenchus brevicomi, a parasite of the southern pine beetle Dendroctonus frontalis. J. Nematol., I980, $12,207-212$.

Martoja R., Martoja M. : Initiation aux techniques de l'histologie animale. Masson et Cie, Paris, 1967,345 .

Massey C. L. : Life history of Aphelenchulus elongatus Massey (Nem.) an endoparasite of Ips confusus Le Conte, with a description of the male. J. Insect Pathol., 1962, 4, 95-103.

MAssey C. L. : Biology and Taxonomy of nematodes parasites and associates of bark beetles in the U.S. - U. S. Dpt. For. Service. A gricultural Handbook, I974, nº 446, 238.

Nickle W. R. : Notes on the genus, Contortylenchus Rühm, I956, with observation on the biology and life history of $C$. elongatus (Massey, 1960 ), n. comb., a parasite of a bark beetle. Proc. Helm. Soc. Wash., 30, 218-223.

Nickle W. R. : Nematode infections. In : Cantwell: Insects diseases, vol. II. Marcel Dekker Publ., New York, 1974, 327-376.

Pornar G. O. : Entomogenous nematodes. A manual and host list of insect nematode associations. E. J. BRILL, Leiden, The Netherlands, I975, 317 .

PoINAR G. O., Hess R. : An ultrastructural study of the response of Blatella germanica (Orthoptera : Blattidae) to the nematode Abbreviata caucasica (Spirurida : Physalopteridae). Int. J. Parasitol., 1974, 4, 133-138.

Rüнм W. : Die Nematoden der Ipiden. Parasitol. SchrReihe, I956, 6, I-437.

Welch H. E. : Entomophilic nematodes. Ann. Rev. Entomol., 1965, 10, 275-302. 